\title{
Hardware Design and Implementation of IP-over-1394 Protocol Stack and Its Evaluation
}

\author{
Kôki Abe* Member \\ Mohd Yusairi Bin Abu Hassan* Non-member
}

\begin{abstract}
This paper describes the hardware design of core functions of the Internet protocol IP over IEEE1394 interface (IP over 1394) and its implementation on an FPGA. The design was evaluated by counting the number of FPGA logic elements required for the implementation. Using a system clock of $49.152 \mathrm{MHz}$, we verified that packets sent from an application on top of the protocol stack were correctly received by the other protocol stack via the IEEE1394 port at a transfer rate of $400 \mathrm{Mbps}$. We also verified the communication behaviors of the design with an isochronous resource manager to reserve a channel prior to data transmissions. The hardware cost of the core IP layer was less than that of the link layer. The evaluation results will help the IP-over-1394 designers explore quantitatively various spectrum of the software/hardware design alternatives.
\end{abstract}

Keywords: IP over IEEE1394, Internet Protocol, IEEE1394, FireWire, hardware implementation, FPGA

\section{Introduction}

It is now commonplace for users at home to set up networks connecting their PCs and peripherals. In addition, more and more audio/video (AV) data are being sent through such networks. Networking AV devices with PCs entails processing, transmitting, and receiving the digital AV data at high speeds. The network should be inexpensive for home use.

Due to its distinctive features such as high-speed data transfers at low cost, plug-and-play functions, etc., the IEEE1394 interface ${ }^{(1)}$, also known as FireWire, has been considered to be a good candidate for physical links in home networks. A standard protocol for the Internet Protocol over the IEEE1394 interface (IP over 1394) has been specified as an RFC (Request For Comment) ${ }^{(2)}$, and preliminary software implementations of the protocol are availabe ${ }^{(3)(4)}$.

Several design choices exist in realizing efficiently IPover-1394 protocol at home networks: (1) to use general purpose processors for entire functions; (2) to use dedicated hardware for functions that constitute the communication bottleneck; (3) to use network processors. The choice (2) will yield a cost-effective implementation for home use. The objective of this paper is to evaluate the cost of the dedicated hardware to alleviate the bottleneck in order to enable the IP-over-1394 designers to explore software/hardware design alternatives.

The bottleneck obviously exists in the core functions of the IP network layer and data-link layer for transferring and processing IP data at high speed using 1394. We evaluate the cost for realizing the core functions of IP over 1394 by hardware.

A hardware implementation of $\mathrm{IPv}_{\mathrm{v}} 4$ has been report-

\footnotetext{
* Department of Computer Science, the University of ElectroCommunications,

1-5-1 Chofugaoka Chofu-shi, Tokyo 182-8585
}

$\mathrm{ed}^{(5)}$, and a reduced set of IPv6 has also been realized in hardware ${ }^{(6)}$. However, these works are based on Ethernet.

We adopt an FPGA as the implementation device and measure the cost by counting the number of FPGA logic elements required for the implementation. The FPGA is useful in that it facilitates prototyping the design, verifying the operations, and evaluating the relative costs among protocol layers. The FPGA realization also gives a lower bound of performance for a hardware implementation.

In the following, after giving an overview of the IEEE1394 features, we will describe the operations of IP over 1394, the design of the hardware modules, implementation results, the experiments for the design verification, and the evaluation of the results.

\section{Features of IEEE1394}

IEEE1394 supports high-speed data transfers of 100 Mbps to $400 \mathrm{Mbps}$. (IEEE1394b enables transfers at a rate of $3.2 \mathrm{Gbps}^{(7)}$.) These speeds are high enough for transferring large packets such as AV data packets.

The IEEE 1394 has a serial bus architecture. Unlike Ethernet, it uses a collision-free medium managed by a full arbiter. The IEEE1394 arbiter realizes a fairness protocol, which prevents the bus from being monopolized by the fastest node; thus all nodes have an equal opportunity to send.

With plug-and-play functions, IEEE1394 supports automatic configuration that allows a new device to be attached or removed from the bus without the need to set or reset the node ID and addresses, a feature different from USB or Ethernet.

\section{Operations of IEEE1394}

In this section, we overview the transactions handled by IEEE1394 and the operations performed by the link 
layer (LLC) and physical layer (PHY) for executing the transactions.

3.1 Transactions For transmitting or receiving IEEE1394 packets, two types of data transfer service, isochronous and asynchronous data transfers, are supported, and a set of transactions are provided for the services. The isochronous transaction transfers data via a channel with a reserved bus bandwidth. An asynchronous transaction called an asynchronous stream packet transfers data via a channel and has the same packet format as the isochronous transaction except that it does not require bus bandwidth. The asynchronous stream packet, also known as the Global Asynchronous Stream Packet (GASP) ${ }^{(8)}$, is used in the IP-over-1394 data transfer, where a channel must be reserved in advanced.

Allocating channels to nodes requesting data transfers must be managed by a central node in an IEEE1394 network. The function is called the isochronous resource manager (IRM), and a node equipped with the function is called an IRM node. A cycle-start packet is broadcast by a node taking the role of the IRM, which can be known from the node ID specified in the packet. In order to reserve a channel, the node must gain a preemptive access to the IRM node. Several transactions provide the mechanism to enable such an access, including the read request/response data quadlet to obtain available channel numbers within an address location in the IRM node.

3.2 Operations by LLC and PHY The IEEE1394 interface consists of link layer (LLC) and physical layer (PHY) as shown in Figure 1, where the flow of data $\mathrm{D}[7: 0]$ are controlled by the bidirectional serial buses Ctlo and Ctl1. The Lreq terminal controlled

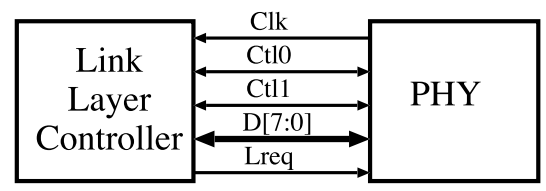

Fig. 1. PHY-LLC interface.

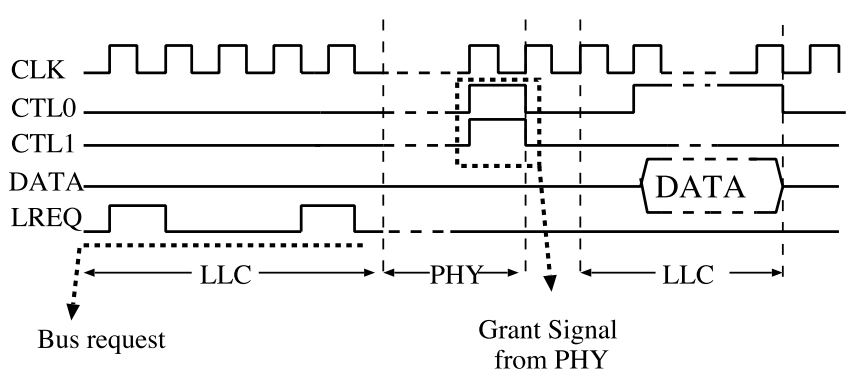

Fig. 2. Transmit operation.

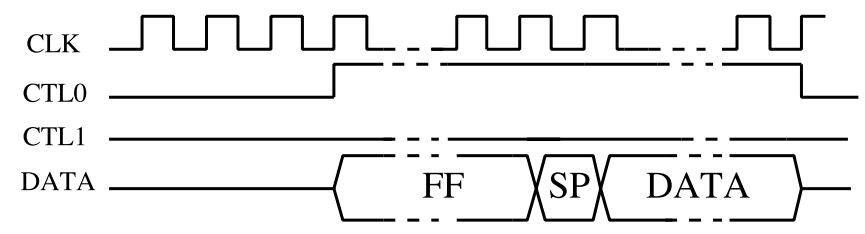

Fig. 3. Receive operation. by LLC sends service requests to PHY in order to gain access to the bus for packet transmission.

Figures 2 and 3 show the timing required for the transmit and receiving operations, respectively. A bus request made by LLC, when granted by a signal from PHY, is followed by sending data packets from LLC. When receiving packets, all bidirectional terminals are controlled by PHY. Incoming packets should start with OxFFs, followed by the speed code $(\mathrm{SP})$ and data.

\section{IP-over-1394 Protocol}

Networking protocols are normally structured in a layered stack ${ }^{(9)}$. A minimal set of operational protocol stack is required in order to estimate the cost of dedicated hardware for the core functions relevant to the bottleneck. The protocol stack we employed for that purpose is shown in Figure 4. The stack includes the UDP (User Datagram Protocol) for the transport layer, IP (Internet Protocol) for the network layer, and IEEE1394 for the data-link layer; the latter two constitute the IPover-IEEE1394. Such protocols as DHCP, RARP, ARP, and ICMP are not included here because they are not relevant to the bottleneck and supposed to be realized by software.

4.1 Transport Layer UDP for the transport layer is employed in this study. Although UDP provides no reliability, it requires minimal resources and overheads for realizing the transport layer.

We note that a kind of reliable data transmission is guaranteed by the mechanism of the IEEE1394 link layer, where a channel between nodes is reserved before transmission. Retry protocols are also provided within the 1394 specifications ${ }^{(2)}$.

4.2 Network Layer The network layer protocol can set and recognize the IP address of where the packet be transmitted for all types of network. We adopt TinyIP ${ }^{(10)}$, a reduced set of IP version 4 , for the network layer. TinyIP is suitable for our purpose in that it provides an end host with the core IP functions required for home networking.

4.3 Data Link Layer We use IEEE1394 for the data link layer. It is reasonable to assume that there would be a node taking the role of a gateway to the Internet in an IP-over-1394 home network. This node would usually be a PC, and we rely on such a PC for the IRM function and do not include the function in the current implementation of the IP-over-1394 module.

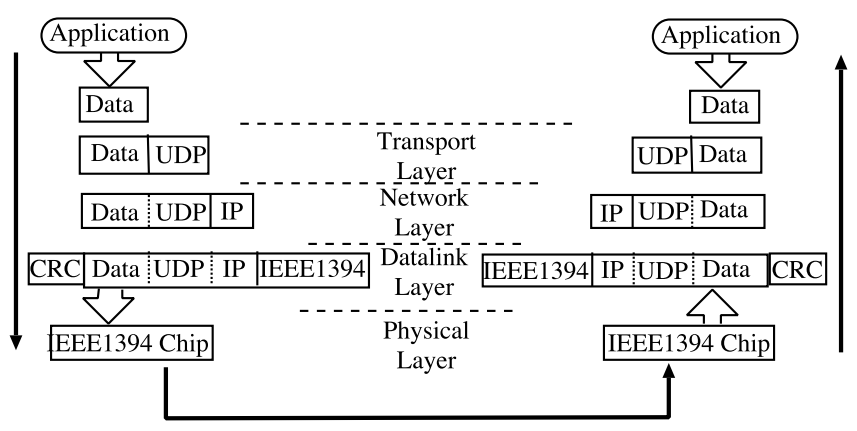

Fig. 4. UDP/IP protocol suite. 
When using the IEEE1394 link header for sending IP datagrams, the protocol specifies that they should be transmitted via asynchronous stream packets which must conform to the GASP format.

\section{Design of IP-over-1394 Module}

A hardware module realizing the core functions of the protocol suite listed in Table 1 was designed. We call the module "fireIP."

The block diagram of fireIP is shown in Figure 5 . It consists of fire_trans and fire_rec for transmitting and receiving data, respectively, and control for establishing the connection between nodes.

The module fire_trans consists of net_trans and link_trans. The net_trans module processes the transport and network layer protocols for data to be transmitted and hands the results to the link_trans module for the link layer protocol. The fire_rec module for receiving data consists of net_rec for the transport and network layers and link_rec for the link layer.

5.1 LLC - PHY interface First we describe the modules controlling the interface between the link and PHY layers.

- fifo: This module is used as a buffer to retain data in the module before transferring them to other modules.

- crc: This module (not shown in Figure5) calculates the CRC (Cyclic Redundant Check) value while

Table 1. Protocols constituting the fireIP.

\begin{tabular}{l|c}
\hline Layer & Protocol \\
\hline \hline Transport & UDP \\
\hline Network & TinyIP \\
\hline Data Link & IEEE1394 GASP \\
\hline
\end{tabular}

transmitting and receiving packets. For receiving, the calculated value is compared with the received CRC. For transmitting, the calculated value is attached to the packet.

- Ireq: This module issues a bus request through the Lreq terminal to get permission to use the bus from PHY.

- trans: This module controls the use of data_out[7:0] according to the speed request. It also controls the transmission timing. The trans module starts transmitting link packets after receiving the grant signal from PHY. When transmitting data to PHY, trans asserts the ctlo_out terminal to request PHY that the data be transmitted.

- rec: The PHY hands the received packet to the module rec by asserting the ctl0_in terminal. The terminals of data_in[7:0] used by the module to receive the packet depend on the transmission speed. The rec module then puts the packet into the fifo and sends a signal to link_rec to inform trans of the reception.

5.2 Link Layer The modules for the link layer protocol deal with two types of asynchronous packet: the asynchronous stream packets are handled by stream_trans and stream_rec; the asynchronous packets are handled by asyn_trans and asyn_rec modules.

- stream_trans: After receiving the signal from ip_trans to start transmission, stream_trans generates a link header, attaches it to the IP data, and puts the data into the fifo of the trans module. The header includes GASP and encapsulation headers. The CRC is calculated twice: once for the header and once for the data. When the packet is ready to be transmitted, a signal is sent to inform the trans module that IP data has been put into the fifo and

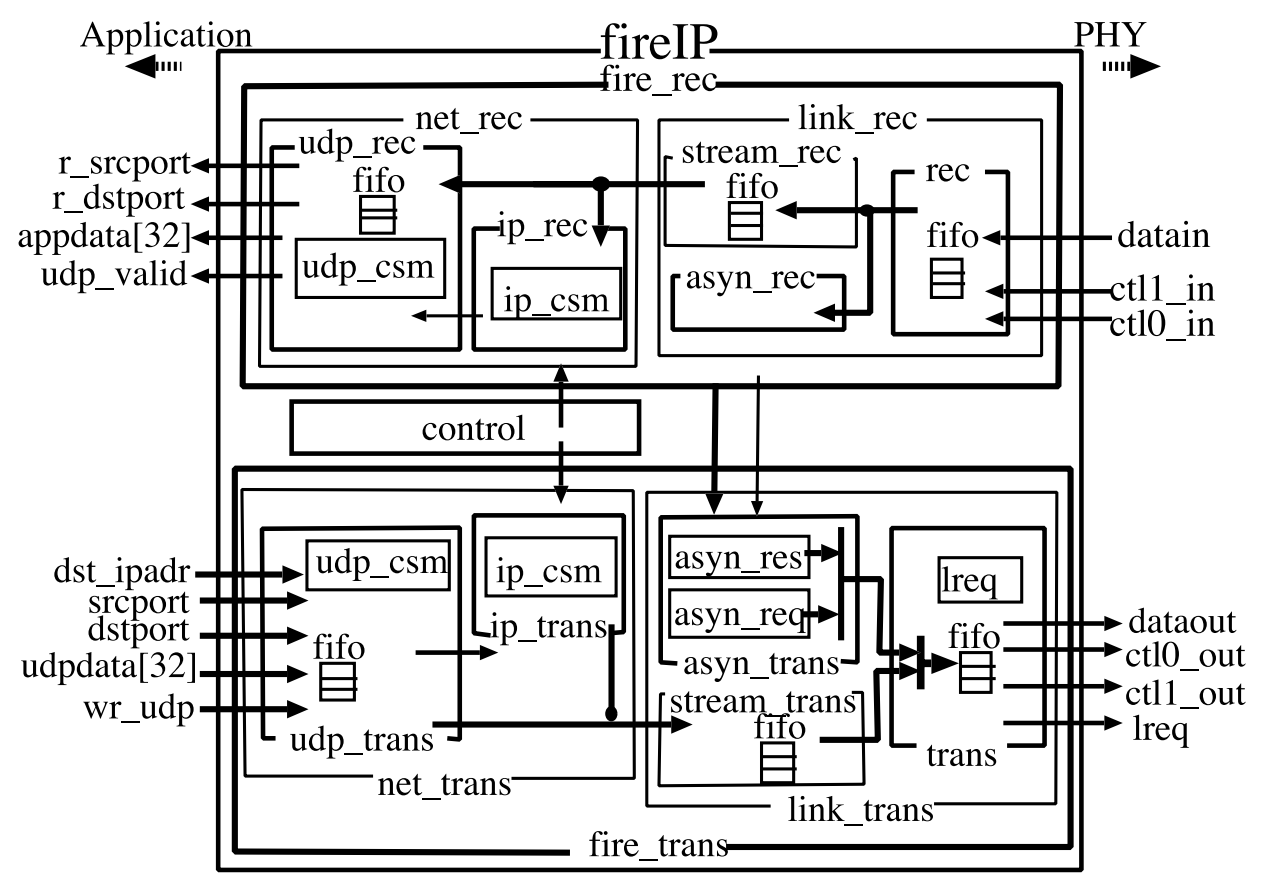

Fig. 5. Block diagram of fireIP module. 
is ready to be transmitted.

- stream_rec: On detecting the signal from rec, stream_rec takes the data from the fifo and checks the link header. If the transaction code is equal to 0xA of the GASP type, stream_rec will start the header check process. Otherwise, the packet will be ignored. The header check process checks the header and data CRCs and the type field value. If both of the CRCs are correct and the type field equals 0x0800 ( $\left.\mathrm{IPv}_{\mathrm{v}} 4\right)$, the packet is accepted and handed to the ip_rec. Otherwise, the packet is discarded and stearm_rec clears the fifo in rec.

- asyn_trans: This module includes two modules, asyn_req and asyn_res, for generating asynchronous request and response packets, respectively.

- asyn_req: This module acts as an asynchronous request packet generator to make requests to another node.

- asyn_res: This module is used to make a response to a request from other node.

- asyn_rec: When receiving a packet containing a transaction code other than 0xA of the stream packet transmission, asyn_rec will process the packet and inform the control about what to do with the packet.

5.3 Network Layer The network layer consists of two modules, ip_trans and ip_rec, for transmitting and receiving the IP datagram, respectively.

- ip_trans: This module starts its process after receiving a signal from the transport layer by generating its own IP address and providing other header fields, including IP checksum. When the data is ready, it is put into the fifo of stream_trans. Then ip_trans sends a signal to stream_trans to inform it that data is ready to be transmitted.

- ip_rec: This module reads its fifo when it detects the ready signal asserted by stream_rec. It also checks the destination address. If the address matches its own IP address, it will accept the packet and calculate the IP checksum. If the calculated value equals the received IP checksum, ip_rec asserts sends a signal to its transport layer's module to inform it of the packet's arrival.

5.4 Transport Layer The modules for the transport layer process data requested by the application to transmit and data received from the network layer. The udp_trans and udp_rec modules are used for transmission and reception, respectively.

- udp_trans: Data generated by an application is put into the fifo of udp_trans module, where a UDP header is attached to the data. The UDP header includes destination and source ports, data length, and UDP checksum. When data with the UDP header is ready, udp_trans sends a signal to ip_trans to inform it that the UDP data is ready to be sent.

- udp_rec: This module checks length, header checksum, port, etc. If matched, the packet is accepted and udp_rec sends a signal to inform the application layer of the packet's reception.

\section{Implementation Method and Results}

Verilog-HDL code totaling about 4,500 lines was developed for the design.

An SRAM based FPGA chip APEX20K- 400EBC652$1 \mathrm{X}^{(11)}$ by Altera was chosen for implementing the design. The FPGA is capable of realizing 400,000-gate logic circuits using Logic Elements (LEs) and 212,992 bit memory using Embedded System Blocks (ESBs). An EDA tool of Quartus II ver.2.0 provided by the FPGA vendor and a logic synthesis tool, Synplify Pro7.1 ${ }^{(12)}$ by Synplicity, were used throughout the implementation.

The Verilog-HDL descriptions were synthesized and converted into netlists by using Synplify, after which they were fitted to the FPGA cells by using Quartus. The compilation results are shown in Table 2, which lists the the LEs and ESBs required for realizing each component module of the fireIP and the maximum operating frequencies (f_max) of the compiled modules. The relative numbers of LEs and ESBs used for each module per total LEs and ESBs available in the FPGA are also shown as percentages.

As shown in Table 2, the maximum frequency of fireIP is $56.41 \mathrm{MHz}$. Because IEEE1394 is specified to operate at $49.152 \mathrm{MHz}$, we can say that the design has enough speed to operate. The module fireIP requires $22 \%$ of the 400,000 gates and uses $19 \%$ of the 212,992 memory bits in the FPGA.

\section{Design Verification Experiments}

To verify the design, two sets of experiments, (1) and (2), were performed. In this section, we describe the experimental environment, the operations to be verified, and the results of the verifications for each experiment.

7.1 Experiment (1) The purpose of this experiment is to verify that proper communications exist be-

Table 2. Compilation results.

\begin{tabular}{l|r|r|r}
\hline Module & LEs* & ESBs* & f_max \\
\hline \hline fire_ip & $3,661(22 \%)$ & $40,960(19 \%)$ & 56.41 \\
\hline fire_trans & $1,394(8 \%)$ & $16,384(7 \%)$ & 71.49 \\
fire_rec & $2,171(13 \%)$ & $24,576(11 \%)$ & 55.49 \\
\hline net_trans & $945(5 \%)$ & $8192(3 \%)$ & 71.35 \\
udp_trans & $510(3 \%)$ & $0(0 \%)$ & 78.08 \\
udp_csm & $364(2 \%)$ & $0(0 \%)$ & 58.84 \\
ip_trans & $303(1 \%)$ & $0(0 \%)$ & 73.89 \\
ip_csm & $255(1 \%)$ & $0(0 \%)$ & 114.98 \\
\hline net_rec & $1,047(6 \%)$ & $8192(3 \%)$ & 56.60 \\
udp_rec & $576(3 \%)$ & $8192(3 \%)$ & 57.90 \\
ip_rec & $460(2 \%)$ & $0(0 \%)$ & 70.04 \\
\hline link_trans & $1,166(7 \%)$ & $16,384(7 \%)$ & 60.91 \\
stream_trans & $404(2 \%)$ & $8192(3 \%)$ & 108.12 \\
asyn_trans & $668(1 \%)$ & $0(0 \%)$ & 81.29 \\
asy_req & $323(1 \%)$ & $0(0 \%)$ & 175.44 \\
asy_res & $169(1 \%)$ & $0(0 \%)$ & 194.25 \\
trans & $150(1 \%)$ & $8192(3 \%)$ & 122.23 \\
lreq & $28(1 \%)$ & $0(0 \%)$ & 263.71 \\
\hline link_rec & $1,200(7 \%)$ & $16,384(7 \%)$ & 59.35 \\
asyn_rec & $531(3 \%)$ & $0(0 \%)$ & 73.45 \\
rec & $397(2 \%)$ & $8,192(3 \%)$ & 112.57 \\
\hline crc & $191(1 \%)$ & $0(0 \%)$ & - \\
fifo & $39(<1 \%)$ & $8192(3 \%)$ & 186.78 \\
\hline
\end{tabular}

*The available LEs and ESBs totaled 16,640 and 212,992 , respectively. 


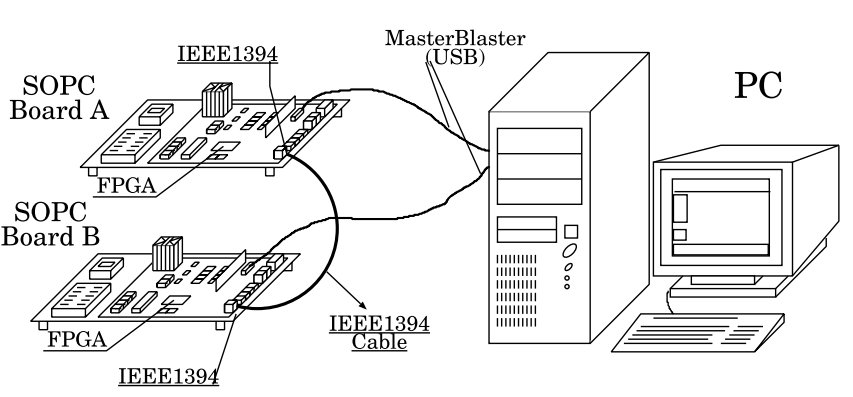

Fig. 6. System used for experiment (1).

tween two fireIP modules.

7.1.1 Experimental Environment The experiment was performed using the system shown in Figure 6. The system consisted of two boards called SOPC boards A and B and a PC (running Windows2000 on a Pentium4 1.7GHz) for monitoring the behavior of the implementation, by using the SignalTap function ${ }^{(13)}$ provided by Quartus with ESBs. The PC was also used for compiling the design and configuring the FPGAs.

Each of the boards was equipped with the FPGA and an IEEE1394 PHY chip TSB41LV03 ${ }^{(14)}$ by Texas Instruments. A 6 pin-to-6 pin IEEE1394 cable and two cables called MasterBlasters were also used. The latter cables were used to configure the FPGAs.

An IP address was allocated to each board: 192.168.0.1 to SOPC board A and 192.168.0.2 to SOPC board B.

\subsubsection{Verified Operations}

- Transmitting packets: A simple module was designed to provide the application data and addresses for triggering the transmission of packets. The application module on SOPC board A generated data (0x610A) and SOPC board B's IP address and passed them to the fireIP module where the data were encapsulated and transmitted to SOPC board B. The outgoing packet was analyzed on board A by using SignalTap.

- Receiving packets: SOPC board B waited for the incoming packet. The received packet was analyzed on board B by using Signal Tap. The received packets decapsulated by fireIP were then compared with the transmitted data.

A $49.152 \mathrm{MHz}$ clock was used for the IEEE1394 system clock, and the transfer rate was $400 \mathrm{Mbps}$. Note that the maximum frequency of the fireIPs was high enough for the clock rate.

7.1.3 Results of Verifications Figures 7 and 8 show a transmitted packet observed at the SOPC board $\mathrm{A}$ and a received packet observed at the SOPC board B. The waveforms were as expected: the data 0x610A reaching the receiver's application layer were the same as those transmitted by the sender's application layer. The values of CRC, IP and UDP checksums were correctly calculated on both sides, and the address check functions of each module also worked well.

7.2 Experiment (2) As explained previously, IP-over-1394 packets use the GASP format, which specifies that a channel number should be reserved for communication between nodes. Before transmitting an IP-

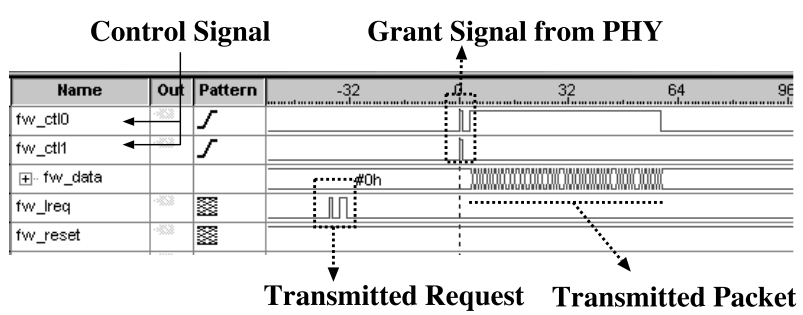

Fig. 7. Waveform observed at SOPC board A in experiment (1).

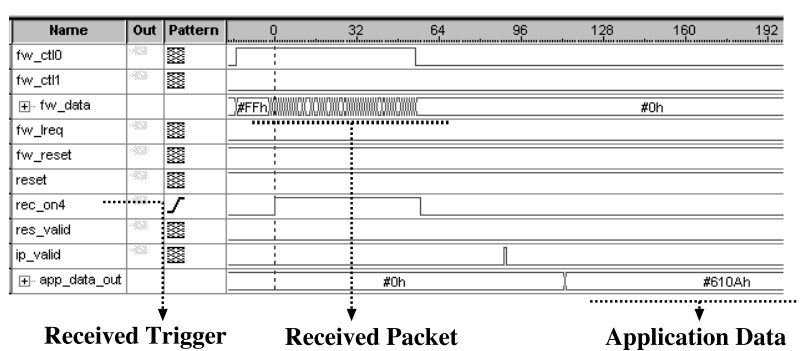

Fig. 8. Waveform observed at SOPC board B in experiment (1).

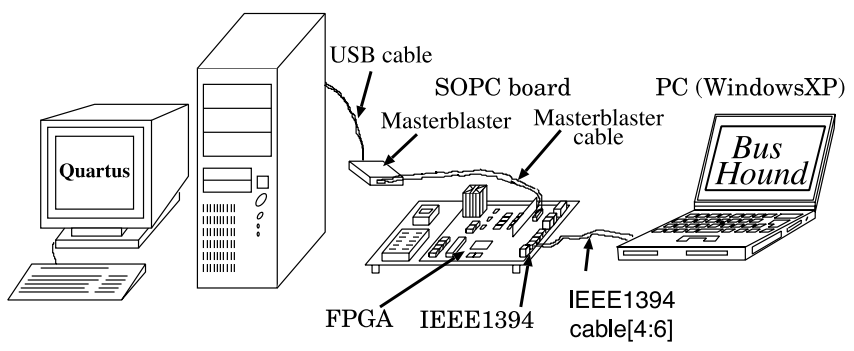

Fig. 9. System used for experiment (2).

over-1394 packet, fireIP needs to reserve a channel from an IRM node. The purpose of this experiment is to verify that fireIP is able to reserve a channel by communicating with the IRM node.

7.2.1 Experimental Environment A set of sub-experiments were performed using the system shown in Figure 9. The system consisted of a PC (WindowsXP) taking the role of the IRM node and an SOPC board implementing the fireIP module. A software tool, Bus Hound $4.0^{(15)}$, was used by the PC to monitor the 1394 packets.

7.2.2 Verified Operations We verified the following operations:

- Obtaining IRM node identifier: As described in section 4.3, the IRM function which manages the channel allocation is assumed to be implemented on a gateway, which was the $\mathrm{PC}$ in this experiment. The IRM node broadcasted a cycle start packet. On receiving the packet, fireIP detected the identifier of the IRM node.

- Obtaining valid channel numbers: By sending a read request data quadlet packet to the address location of the IRM node, valid channel numbers could be obtained.

- Reserving a channel: A channel number must be locked in order to reserve the channel. The lock operation started by sending a lock request packet 


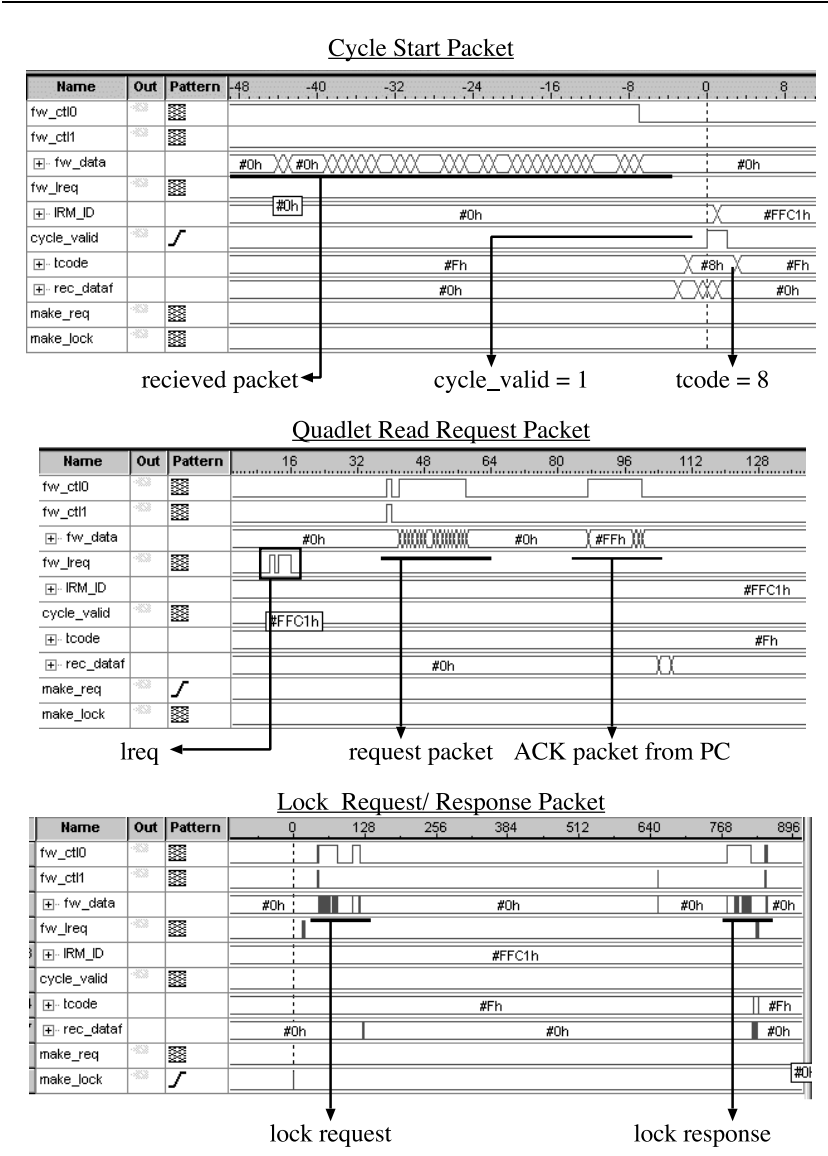

Fig. 10. Waveforms observed at the SOPC board in experiment (2).

to the IRM node. If a lock response packet was responded with no error, the lock operation ended successfully.

7.2.3 Results of Verifications Figure 10 shows the waveforms observed in the experiments. At the bottom we see that the lock operation ended successfully by properly receiving a lock response packet. . The cycle start packet from the IRM node notified the IRM node identifier. Furthermore, the channel selected from valid ones indicated in the quadlet data sent in response to the read request packet was found to be reserved by lock request/reply transactions. We therefore verified all the operations required for reserving a channel used for transmitting IP-over-1394 packets.

\section{Evaluation and Discussion}

As shown in Table 2, the maximum frequency f_max of fireIP is higher than $49.152 \mathrm{MHz}$, which corresponds to $400 \mathrm{Mbps}$, the upper limit of 1394's throughput. Thus the processing speed of the module is higher than the communication speed of the 1394 medium, meaning that the performance of the implementation meets the requirements.

The hardware cost in terms of number of FPGA logic elements of the IEEE1394 link layer is 2,366, while total cost of the network, transport, and application layers is 1,992 . This means that the upper layers' cost is $84 \%$ of the link layer's. That is, when implementing the core functions of IP over 1394 with hardware, the additional cost does not exceed the cost of the IEEE1394 link layer part, which is normally realized with hardware.

Inexpensive embedded processors are widely available, which could be used for realizing the IP-over-1394 protocol. However, the high-speed processing required for the network and data link layers would overload the processor. The additional cost estimated for the dedicated hardware to remove the bottleneck will well be justified. The evaluation results will help the IP-over-1394 designers explore quantitatively various spectrum of the software/hardware design alternatives.

Although the descriptions of the hardware design described in this paper were synthesized and mapped onto an FPGA, they can easily be retargeted to an ASIC. The recent progress in semiconductor technology enables larger number of gates to be fabricated on a chip. In such circumstances, the IP-over-1394 stack can be embedded as a functional module in a system-on-a-chip.

We measured the hardware cost by counting the number of required logic elements in the FPGA. Although an FPGA is suitable for prototyping a design, FPGA realizations are generally slower and larger than ASIC realizations. An ASIC version of the current IP-over-1394 design would yield an implementation with higher speed and lower gate counts. The IEEE1394 serial bus has been revised to support a transfer rate of $3.2 \mathrm{Gbps}^{(7)}$. It will be possible to obtain a chip operating at this speed if the design described in this paper is realized as an ASIC.

Such protocols as DHCP, ARP, and ICMP for initializing the data transfer and processing errors are irrelevant to the bottleneck, which could be realized more efficiently by software than by hardware. It is reasonable to ensure the interoperability with existing devices like home routers by software.

\section{Conclusions}

The cost of dedicated hardware required for realizing the core functions of IP-over-1394 protocol was evaluated. Using a system clock of $49.152 \mathrm{MHz}$, we verified that packets sent from an application on top of the protocol stack were correctly received by the other protocol stack via the IEEE1394 port at a transfer rate of $400 \mathrm{Mbps}$. We also verified the communication behaviors of the design with an isochronous resource manager to reserve a channel prior to data transmissions. The hardware cost of the core IP layer was less than that of the link layer. The evaluation results will help the IPover-1394 designers explore quantitatively various spectrum of the software/hardware design alternatives.

Communications with software versions of IP over1394 have not been confirmed yet. Software implementations of IP over 1394 differ among operating systems ${ }^{(3)(4)}$. This implies that the standardization of the protocol should further be scrutinized.

We recognize the importance of using IPv6 in IP-over1394. Real-time protocols such as RTP/RTCP at transport and higher layers are useful for AV data transfer. Evaluating the cost of processing such protocols by hardware belongs to future works. 


\section{Acknowledgment}

The authors would like to express our thanks to Dr. Masachika Harada and Dr. Atsushi Takegami with Texas Instruments Japan Ltd., who provided us with valuable information about the PHY chip. They also would like to thank Dr. Takamichi Tateoka with the Department of Computer Science at the University of Electro-Communications for his important suggestions and discussions on the IP protocol. Finally, they would like to thank the anonymous reviewers for their helpful comments to improve the quality of the manuscript.

(Manuscript received Dec. 10, 2003,

revised 10, 2004)

\section{References}

(1) IEEE Standards Board: "IEEE Standard for a High Performance Serial Bus", IEEE Std 1394-1995 (1995)

(2) P. Johansson: "IPv4 over IEEE1394", RFC2734 (1999)

( 3 ) Open Source Development Network, http://www.linux1394.org (2002)

(4) Microsoft Co., http://www.microsoft.com (2002)

(5) K. Morita and K. Abe: "Implementation of UDP/IP Protocol on FPGA and Its Performance Evalution", Proc. IPSJ General Conf., Special 5, pp.157-158 (2001)

(6) Y. Izuhara, K. Morita, T. Tateoka, and K. Abe: "Specification of TinyIPv6 Protocol Stack for Remote Control and Its Implementation on FPGA", IPSJ Journal, Vol.43, No.11. pp.3540-3548 (2002)

(7) IEEE Standards Board: "IEEE Standard for a High Performance Serial Bus - Amendment 1", IEEE Std 1394b-2002 (2002)

(8) IEEE Standards Board: "IEEE Standard for a High Performance Serial Bus - Amendment 1", IEEE Std 1394a-2000 (2000)

(9) W. R. Stevens: TCP/IP Illustrated, Vol.1: The Protocol, Addison-Wesley (1994)

(10) T. Tateoka and K. Abe: "Design and Implementation of
TinyIP: A Restricted UDP/IP Stack for Educational Use", Trans. IEICE B, Vol.J86-B, No.8, Aug., 2003, To appear.

(11) Altera Co., APEX 20K Programmable Logic Device Family, http://www.altera.com/literature/lit-apx.html, 2002.

(12) Synplicity Inc., Synplify Pro 7.1 Release Notes, http://www.synplicity.com/literature/index.html, 2002.

(13) Altera Co., SignalTap Analysis in the Quartus II Software Ver.2.0,

http://www.altera.com/literature/an/an175.pdf, 2002.

(14) Texas Instruments Inc., TSB41LV03 IEEE 1394a Three Port Cable Transceiver/Arbiter, 1998.

(15) Perisoft, http://www.perisoft.net/bushound.

Kôki Abe (Member) received the B.E. and M.E. degrees

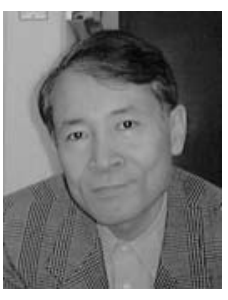
from Yokohama National University, Japan, in 1969 and 1971, respectively, and the Ph.D. degree from the University of Tokyo, Japan, in 1976. Since 1974 he has been with the University of Electro-Communications, Tokyo, Japan, where he is currently an associate professor of the Department of Computer Science. From 1980 to 1982, he was a visiting researcher at Carnegie-Mellon University, PA. USA. His current teaching/research interests include computer architecture, VLSI design, and computer networking. Dr. Abe is a member of the IEEE Computer Society, the Information Processing Society of Japan, and the Institute of Electronics, Information and Communication Engineers.

Mohd Yusairi Bin Abu Hassan (Non-member) received

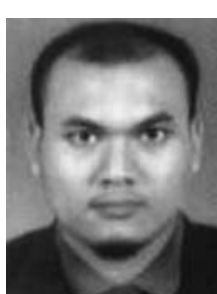
the B.E. and M.E. degrees from the University of Electro-Communications, Tokyo, Japan, in 2001 and 2003 respectively. Since 2003 he has been with Telekom Malaysia, where he is currently engaged in development of network software. 